\title{
Formation of Substellar Objects: A SIRTF Legacy
}

\author{
C. H. Young \& K. M. Guenthner \\ Department of Astronomy, University of Texas at Austin, Austin, TX, \\ 78712
}

\begin{abstract}
We present a summary of SIRTF capabilities and the observing strategy for the SIRTF Cores to Disks Legacy Project.
\end{abstract}

\section{Introduction}

SIRTF, the Space InfraRed Telescope Facility, is expected to be launched in January 2003. This telescope, the final mission of NASA's Great Observatories Program, will provide unsurpassed sensitivity and resolution at mid- to far-infrared wavelengths. Early in its lifetime, SIRTF will complete six Legacy programs aimed at answering some of the most important questions in astronomy. The Legacy left behind by these projects will be their vast data sets that will be publicly available, with no proprietary period. The Legacy team From Molecular Cores to Planet-forming Disks (Cores to Disks, c2d) is pursuing one of these projects. We present the observing strategy for the $c 2 d$ program and investigate the possible implications of the data on understanding the formation of young, substellar objects.

\begin{tabular}{ccc}
\multicolumn{2}{c}{ Table 1. } & \multicolumn{2}{c}{ Sensitivity \& Resolution } \\
\hline$\lambda(\mu \mathrm{m})$ & $3-\sigma(\mathrm{mJy})$ & Resolution $\left({ }^{\prime \prime}\right)$ \\
\hline 3.6 & 0.02 & 2 \\
4.5 & 0.03 & 2 \\
5.8 & 0.08 & 2 \\
8.0 & 0.1 & 6 \\
24 & 1.0 & 6 \\
70 & 5.0 & 17 \\
\hline \hline
\end{tabular}

\section{Observations}

As part of the Cores to Disk Legacy project, SIRTF will observe over 20 square degrees in nearby molecular clouds, regions where substellar objects are possibly forming. These clouds include Chamaeleon II, Lupus, Perseus, Rho Ophiuchus, and Serpens and will be observed with sensitivity and resolution as in Table 1. In addition to the mid- and far-infrared observations with SIRTF, the c2d Legacy team will also deliver millimeter maps of these regions. Additionally, the $c 2 d$ team will observe over 120 star-forming cores spanning the evolutionary sequence from preprotostellar cores to those with infrared sources. These cores are known or candidate sites of star formation and may also be forming young, 


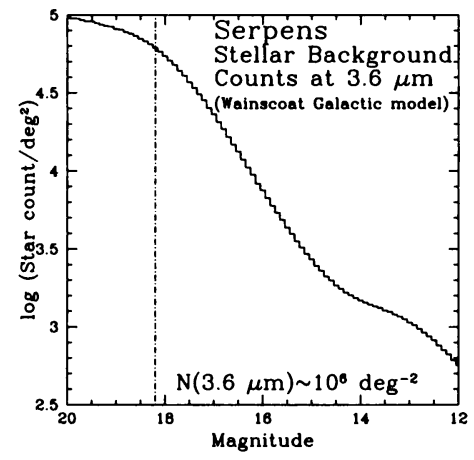

Figure 1. The expected background stellar count observed towards Serpens. With SIRTF sensitivities at $3.6 \mu \mathrm{m}$, we expect to detect about $10^{6}$ background stars.

substellar objects. The complete source list is available on the c2d Web site, http: //peggysue.as .utexas .edu/SIRTF/.

\section{Background Sources}

We expect to observe many new and exciting objects in the course of this work, but, with SIRTF's unprecedented sensitivity, we also expect to detect many background galactic and extra-galactic objects. With a Galactic model (Wainscoat et al. 1992), we have estimated the expected stellar counts towards all five molecular clouds. Serpens, at low galactic latitude, has the largest count with about $10^{6}$ background stars per squ, :e degree (see Figure 1). Additionally, we can expect to detect many thousands of extragalactic background sources within our $5^{\prime} \times 5^{\prime}$ field-of-view (e.g., Dole et al. 2000).

\section{Substellar Objects}

SIRTF will provide the means to understand the processes by which substellar objects form. Do they form in the same way as their stellar colleagues? Natta \& Testi (2001) found the SED of one object to be consistent with a $50 \mathrm{M}_{J u p}$ object surrounded by a $30 \mathrm{M}_{\text {Jup }}$ disk. Indeed, it does seem that some processes in the formation of stellar objects also occur in the formation of brown dwarfs. Within the molecular clouds and cores observed as part of the $c 2 d$ program, we expect to reveal these young, forming brown dwarfs.

\section{References}

Dole, H. et al., 2000, astro-ph/0002283

Natta, A., \& Testi, L., 2001, A\&A, 376, L22

Wainscoat, R.J., et al., 1992, ApJS, 83, 111 